\title{
Conjunctival epithelium expression of HLA-DR in glaucoma patients and its influence on the outcome of filtration surgery
}

\author{
A Ihan, B Cvenkel
}

\begin{abstract}
Aims-To analyse the expression of HLA-DR on conjunctival epithelial cells in patients with glaucoma taking topical antiglaucoma therapy.

Methods-10 patients taking no topical medication and 30 patients with uncontrolled glaucoma taking medical therapy participated in the study. The specimens were obtained by impression cytology preoperatively, 3 months, and 6 months after filtration surgery. The expression of HLA-DR on T lymphocytes and epithelial cells was analysed by flow cytometry.

Results-A significant increase in HLA-DR on epithelial cells was found preoperatively in patients with glaucoma. A significant increased expression of HLA-DR on epithelial cells was detected 3 months and 6 months after surgery.

Conclusions-The increased expression of HLA-DR on conjunctival epithelial cells still present 6 months after surgery indicates the increased ability of epithelial cells to induce immune inflammation with subsequent fibrosis.

(Br F Ophthalmol 2000;84:648-650)
\end{abstract}

Several clinical studies have reported significantly higher success rates of trabeculectomy in patients undergoing primary trabeculectomy than in patients taking topical glaucoma therapy for at least 1 year preoperatively. ${ }^{12}$ Failure of filtering procedures is connected to an increased conjunctival infiltration of fibroblasts and inflammatory cells. ${ }^{34}$ An increased expression of HLA-DR molecules by conjunctival epithelial cells was observed in immunohistochemical analysis. ${ }^{5}$

For better examination of conjunctival epithelium at the cellular level, a quantitative and reliable method is needed. We report the use of impression cytology and flow cytometry to determine the expression of HLA-DR molecules by conjunctival epithelial cells as well as by infiltrating $\mathrm{T}$ lymphocytes.

7, 1000 Ljubljana,

Slovenia

A Ihan

Medical Center Ljubljana, Eye Clinic, Zaloska 2, 1000

Ljubljana, Slovenia B Cvenkel

Correspondence to: B Cvenkel

barbara.cvenkel@kclj.si

Accepted for publication 27 January 2000 taking at least two medications (mean 2.4 (0.5)) were referred for filtration surgery. They had used topical treatments for a mean period of 4.0 (6.9) years (range $0.01-27$ ). The control group included 10 age matched patients (13 eyes) with cataract taking no topical medi- cation. In none of the patients was an ocular surface disorder or inflammation present as evaluated by slit lamp examination, tear break up time, and Schirmer's test.

All patients underwent a Cairns type trabeculectomy. ${ }^{6}$ In all but three cases of chronic angle closure glaucoma a fornix based conjunctival flap was used. All patients received postoperative topical $0.1 \%$ dexamethasone at least four times daily for 2 months, and three times daily for 1 month.

The follow up for all the patients was at least 6 months. The patients were seen on the first postoperative day, at 1 week, at 1 month, 3 months, and then every 3 months. The complete surgical success was defined as an intraocular pressure (IOP) of $21 \mathrm{~mm} \mathrm{Hg}$ or less without medication, and failure as an IOP greater than $21 \mathrm{~mm} \mathrm{Hg}$ with or without medication. Partial success was defined as an IOP pressure of $21 \mathrm{~mm} \mathrm{Hg}$ or less with additional medication.

METHODS

The collection of conjunctival cells was performed by the imprinting of the ocular surface onto Millipore filter paper (VS $0.22 \mu \mathrm{m}$ pore size) after topical anaesthesia with 1-2 drops of tetracaine (amethocaine). Strips of filter paper approximately $3 \times 6 \mathrm{~mm}$ were pressed firmly with a glass rod on the ocular surface for $2 \mathrm{sec}-$ onds and then removed and put in $1 \mathrm{ml}$ of phosphate buffered saline. Two imprints (samples) were obtained from each quadrant (superior, inferior, temporal, and nasal) - that is, gathering eight samples from one eye. The specimens were obtained preoperatively, and then 3 months and 6 months after the procedure.

Monoclonal antibodies conjugated with fluorochromes: CD3 (PerCP), anticytokeratin (PE), and HLA-DR (FITC) antibodies were purchased from Becton Dickinson (Mountain View, CA, USA). Cells were labelled with a specific panel of monoclonal antibodies.

Flow cytometric analysis was performed using a fluorescence activated cell sorter (FACSort, Becton Dickinson). A three parameter analysis was performed to determine the expression of HLA-DR molecules on CD3+ cells ( $\mathrm{T}$ lymphocytes) and cytokeratin positive cells (conjunctival epithelial cells) (Fig 1).

The Student's $t$ test was used to compare the cell counts and their level of HLA-DR fluorescence during follow up.
Results and discussion

Preoperatively, significantly increased ( $p$ $<0.01$ ) expression of HLA-DR molecules on 

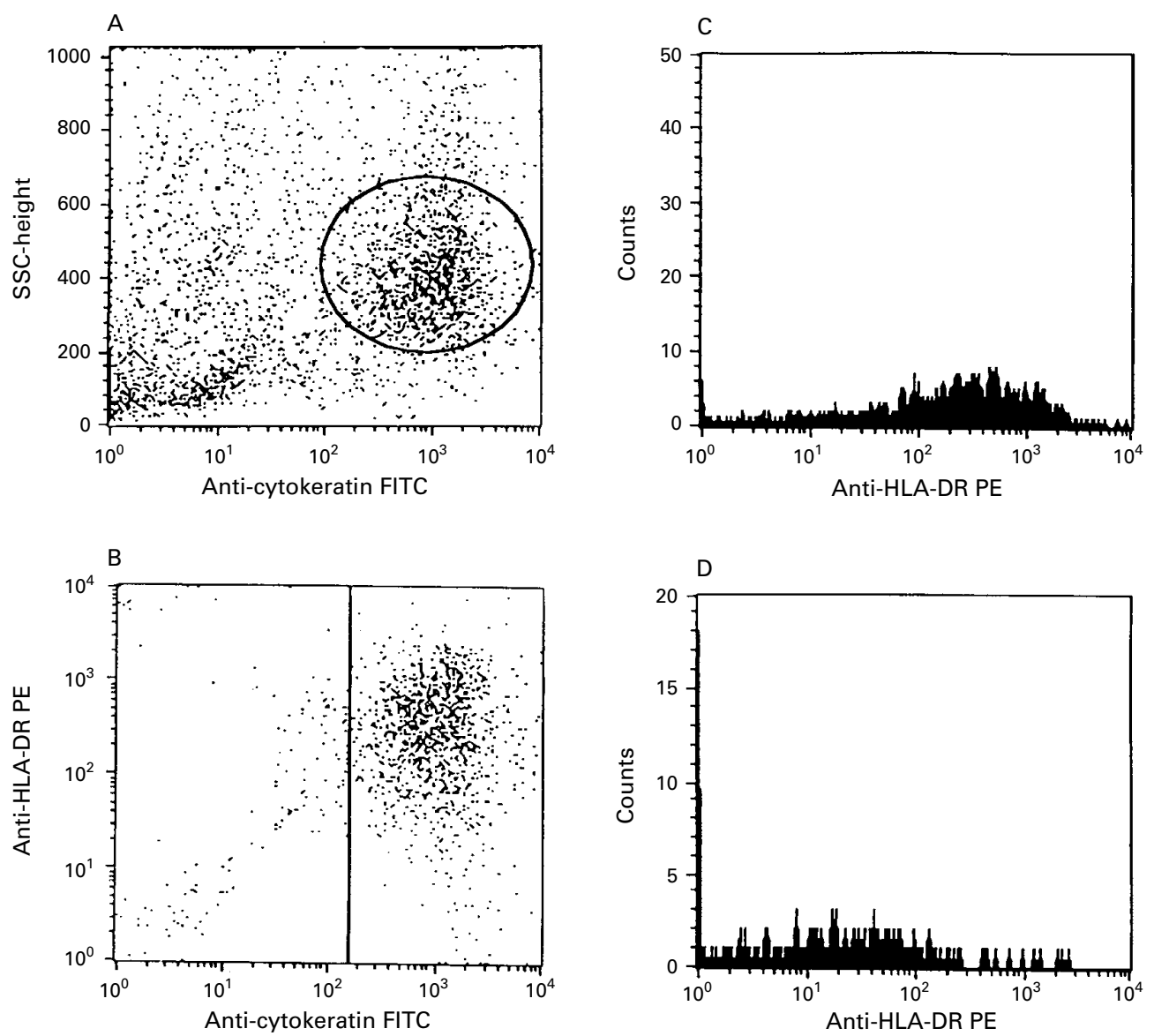

Figure 1 Cells isolated from imprint of ocular surface were incubated with a combination of CD3, anticytokeratin antibodies, and anti-HLA-DR antibodies. Cells were gated on a forward scatter cell size (FCS) versus side scatter (SSC) plot. CD3 positive cells and cytokeratin positive cells were separately analysed for the anti-HLA-DR molecule expression. (A) Epithelial cells were gated on a FSC versus SSC plot. A gate was set in to obtain HLA-DR positive epithelial cells. (B) Double gated cells were analysed for the HLA-DR molecule expression. (C) Epithelial cells with higher mean fluorescence intensities. (D) Epithelial cells with weaker mean fluorescence intensities.

epithelial cells in glaucoma patients versus control group was documented (640.23 (SD 403.29) v 314.00 (156.43)). No significant correlation was found between the duration of topical antiglaucoma therapy and the expression of HLA-DR molecules on epithelial cells and $\mathrm{T}$ lymphocytes before surgery (Pearson correlation coefficient). The percentage of reactive $\mathrm{T}$ lymphocytes and epithelial cells as well as the level of HLA-DR fluorescence by T lymphocytes and epithelial cells was highest 3 months after the operation (Table 1). This finding can be explained by the hypercellular response of normal wound healing being more intense in the first postoperative months. In this period the majority of trabeculectomy failures also occur. ${ }^{7}$ At 6 months the percentage of reactive $\mathrm{T}$ lymphocytes and epithelial cells and the level of HLA-DR fluorescence of T lymphocytes returned to preoperative values

Table 1 Percentage of HLA-DR positive T lymphocytes and epithelial cells and the level of their HLA-DR fluorescence

\begin{tabular}{lccc}
\hline & Preoperative & 3 months & 6 months \\
\hline \% HLA-DR CD3+ & $10.9(10.0)$ & $14.2(13.6)$ & $10.9(9.8)$ \\
HLA-DR expression of CD3+ & $123.8(184.4)$ & $304.6(479.2)$ & $197.5(289.9)$ \\
\% HLA-DR + epithelial cells & $57.5(25.6)$ & $59.8(23.9)$ & $45.8(24.7) \dagger$ \\
HLA-DR expression of epithelial cells & $640.2(403.3)$ & $987.7(668.4)^{\star}$ & $987.7(693.9)^{\star}$ \\
\hline
\end{tabular}

${ }^{\star}$ Statistically significant increase $v$ preoperative $\mathrm{p}<0.05$.

†Statistically significant lower $v 3$ months after surgery $\mathrm{p}<0.05$. which indicates the resolution of immune inflammation (injury, surgery induced inflammation). This finding may correspond to the scar formation (the end of wound healing process) with clinically evident regression of conjunctival hyperaemia.

At 3 months the operation was successful (IOP $\leqslant 21 \mathrm{~mm} \mathrm{Hg}$ without medication) in 26 patients $(86.7 \%)$, at 6 months in 28 patients $(96.2 \%)$. The three failures out of four, at 3 months' follow up, were due to encapsulated filtering blebs and two of the encapsulated blebs underwent spontaneous cystic degenerations, with IOP lowering at 6 months. All failures had IOP more than $21 \mathrm{~mm} \mathrm{Hg}$ with one medication. No significant difference was found in HLA-DR levels between the patients with successful surgery and those with failure, but the very small group of failed patients could explain the absence of apparent predictive effect of preoperative inflammation. In our study the increased expression of HLA-DR by epithelial cells persisted 6 months after surgery although the patients had not been receiving any eye drops for at least 2 months. This may indicate the increased ability of epithelial cells to present antigens to $\mathrm{T}$ lymphocytes and induce immune inflammation to a variety of exogenous antigens. Therefore it may be 
expected that increased expression of HLA-DR molecules by epithelial cells indicate a higher risk for long term immune inflammation with subsequent fibrosis and filtration failure.

Although the majority of trabeculectomy failures occur in the first 3 months after operation, the failures also occur at a slower rate 1 year after surgery. It was reported that if the operation was successful at 1 year, the probability of successful control of IOP was $67 \%$ at 10 years. $^{8}$ There is a continuous scarring present at the site of injury.

Flow cytometry is a new, sensitive, and objective tool for assessing the activation of lymphocytes and inflammatory response of epithelial cells. Combined with impression cytology it enables us to follow the dynamics of ocular surface changes. Longer follow up on a greater number of patients is necessary to clarify whether an increased level of expression of HLA-DR molecules by conjunctival epithelial cells indeed increases the risk for chronic inflammation and related complications with resultant bleb failure.

1 Lavin MJ, Wormald RP, Migdal CS, et al. The influence of prior therapy on the success of trabeculectomy. Arch Ophthalmol 1990;108:1543-8.

2 Broadway DC, Grierson I, O'Brien C, et al. Adverse effects of topical antiglaucoma medication II. The outcome of filtration surgery. Arch Ophthalmol 1994;112:1446-54.

3 Hitchings RA, Grierson I. Clinico-pathological correlation in eyes with failed fistulizing surgery. Eye 1983;103:84-6.

4 Skuta GL, Parrish RK, Wound healing in glaucoma filtering

5 Burgery. Surv Ophthalmol 1987;32:149-70. inflammatory markers by conjunctival cells in chronically treated patients with glaucoma. Ophthalmology 1994;101: treated pati

6 Cairns JE. Trabeculectomy: preliminary report of a new method. Am f Ophthalmol 1968;66:673-9.

7 Inaba Z. Long-term results of trabeculectomy in the Japanese: an analysis of lifetable method. fpn f Ophthalmol 1982;26:361-73.

8 Chen TC, Wilensky JT, Viana MAG. Long-term follow-up of initially successful trabeculectomy. Ophthalmology 1997; 104:1120-5. 